\title{
AN EDGE-BASED MEDIAN FILTERING ALGORITHM WITH CONSIDERATION OF MOTION VECTOR RELIABILITY FOR ADAPTIVE VIDEO DEINTERLACING
}

\author{
Qian Huang $^{1,2}$, Wen Gao ${ }^{1,2}$, Debin Zhao ${ }^{1}$, Qingming Huang ${ }^{2}$ \\ ${ }^{1}$ Institute of Computing Technology, Chinese Academy of Sciences, Beijing 100080, China \\ ${ }^{2}$ Graduate School of the Chinese Academy of Sciences, Beijing 100039, China \\ \{qhuang,wgao, dbzhao, qmhuang\}@jdl.ac.cn
}

\begin{abstract}
Because of its ability to preserve signal edges while filtering out impulsive noises, median filtering is widely used in signal processing applications, e.g. deinterlacing. An edgebased median filtering (EMF) algorithm is proposed for adaptive deinterlacing. A criterion of motion vector reliability (MVR) is also introduced for better interpolation. For each motion compensated block, two motion vectors between opposite-parity fields and one between same-parity fields are taken into account. Experiments show that the proposed MVR and EMF are both very efficient. Outputs of the proposed EMF are much more similar to original progressive videos than those of objectively best EMF methods nowadays, without obvious visual distortions. Finally, the proposed EMF and MVR criterion are shown to be suitable for texture-based adaptive deinterlacing.
\end{abstract}

\section{INTRODUCTION}

Deinterlacing is widely used to reduce the visual artifacts in current TV systems. During the past thirty years, various deinterlacing techniques have been proposed, among which median filtering is particularly popular because of its ability to preserve signal edges while filtering out impulses [1]. Since median filter has the drawback of distorting vertical details and introducing alias [2], edge-based median filtering (EMF) algorithms with motion compensation are more often used. However, motion compensated (MC) EMF methods are for display purpose; hence they cannot reach a good balance between subjective and objective effects. Usually it is meaningless to focus on smoothing video sequences while taking the risk of loosing details, because EMF cannot generate excellent deinterlacing outputs by itself and often acts as one of the candidates in adaptive deinterlacing schemes [3]. Under this consideration, it might be more important to make full use of pixels along motion trajectories for better objective results, thus motion vector reliability (MVR) should also be taken into consideration.
In [3] we have defined a field-based motion detection, which has in total four possible output states, i.e. stationary, horizontally moving, vertically moving, and undirectionally moving. The motion detection utilizes motion vectors between opposite-parity fields, however, same-parity motion vectors are sometimes more reliable, especially for the latter two states. Therefore, both kinds of motion vectors should be considered for each MC block, and a MVR criterion is needed to select the most reliable one for deinterlacing.

In this paper, we first introduce a MVR criterion after field-based motion estimation (ME). Two motion vectors between opposite-parity fields and one between same-parity fields are measured for each MC block; only the most reliable one is reserved. Then we propose an EMF algorithm based on the analysis of MVR, using directional correlations in sub-pixel precision. Experimental results show that we can get even better objective results than objectively best EMF approaches nowadays, while no obvious visual distortions can be observed. We also show that the proposed EMF and MVR criterion are both efficient for texture-based adaptive deinterlacing.

The rest of this paper is organized as follows. Section 2 presents the proposed EMF method based on MVR analysis. Section 3 shows and discusses experimental results. Finally, we draw our conclusion in Section 4.

\section{EMF FOR ADAPTIVE DEINTERLACING}

The EMF to be proposed is a spatio-temporal MC algorithm, in which both intra-field and inter-field pixels are used. Inter-field pixels along motion trajectories are of more importance since our main goal is to get better objective results. Thus a MVR criterion is needed to guarantee the EMF performance.

\subsection{MVR CRITERION}

MVR was previously measured when motion vectors for deinterlacing were finally determined [4]. We introduce a MVR criterion to determine the final motion vector for each 


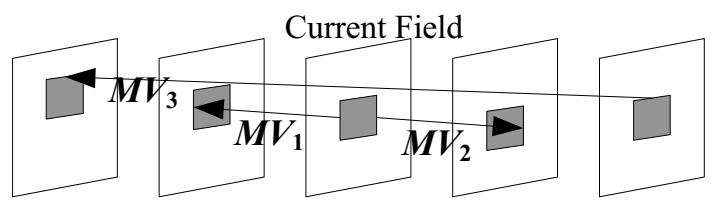

Fig. 1 Fields Used for Motion Vector Determination

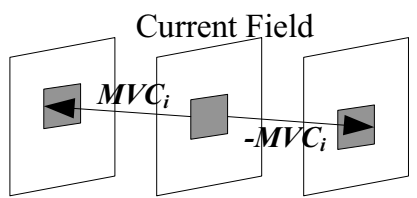

Fig. 2 Motion Vector Candidate after Mapping

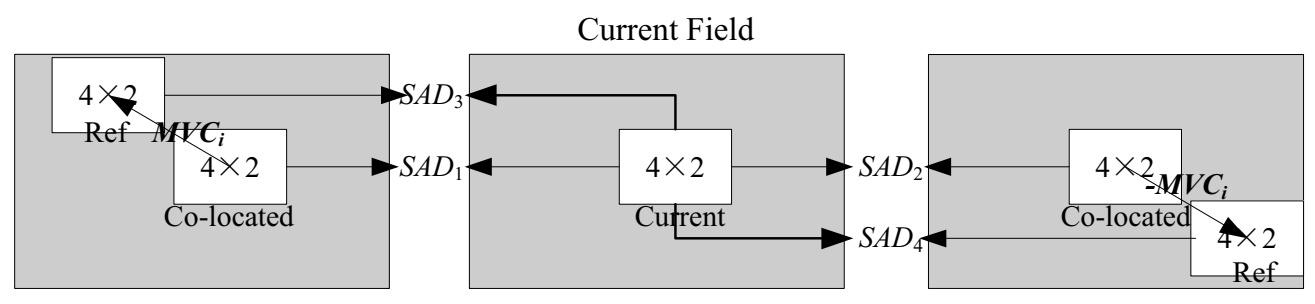

Fig. 3 Calculation of the MVR Factor

MC block after the above-mentioned two kinds of ME, since the field-based ME used in [3] is well-performed. In total five successive fields are used to determine the final motion vector for the current MC block, as illustrated in Fig. 1, where $M V_{1}$ and $M V_{2}$ are motion vectors between opposite-parity fields while $M V_{3}$ is a same-parity motion vector of the co-located MC block in the next same-parity field. Note that we assume ME as well as the sub-block refinement used in [3] has already been performed here.

In order for comparison, each of the three motion vector candidates $M V_{i}(i=1,2,3)$ should first be mapped as a motion vector candidate $M V C_{i}$ of the current MC block:

$$
M V C_{i}=(-1)^{i+1} \cdot M V_{i},
$$

with $M V C_{i}$ the forward motion vector candidate between opposite-parity fields, as depicted in Fig. 2, where the motion vector consistency is assumed.

Then the MVR factor $\alpha$ is defined below to choose the most reliable motion vector. The motion vector candidate that generates the largest $\alpha$ will be selected.

$$
\alpha=\frac{S A D_{1}+S A D_{2}}{S A D_{3}+S A D_{4}},
$$

where $S A D_{1}, S A D_{2}, S A D_{3}$, and $S A D_{4}$ are block-based sums of absolute difference, as shown in Fig. 3.

The average of $S A D_{1}$ and $S A D_{2}$ is in inverse proportion to the similarity of co-located MC blocks. And the average of $S A D_{3}$ and $S A D_{4}$ is in inverse proportion to the similarity of MC blocks along motion trajectory. Therefore, the larger $\alpha$ is, the more reliable the motion vector candidate is.

\subsection{EMF FOR ADAPTIVE DEINTERLACING}

The proposed EMF algorithm uses directional correlations in sub-pixel precision, as shown in Fig. 4, where $f_{n}$ represents the current field and $(x, y)$ designates the spatial position. For the pixel to be interpolated, we consider 9 possible edge directions, among which four are sub-pixel directions. In our algorithm, the sub-pixel values are calculated using a linear interpolation. For example, the value of $A$ is the average of $f_{n}(x-1, y-1)$ and $f_{n}(x, y-1)$.

A zigzag search started from the vertical direction is employed to detect the edge. The search order can be seen from numbers in Fig. 4. At the end of the zigzag search, the direction with minimal absolute difference will be reported as an edge.

Note that sub-pixel values are not original. In order to minimize mistakes, we use the first horizontal differences to exclude impossible sub-pixel directions. Take point $A$ for example, the first horizontal difference is defined as:

$$
\Delta_{x} A=\left|f_{n}(x, y-1)-f_{n}(x-1, y-1)\right| .
$$

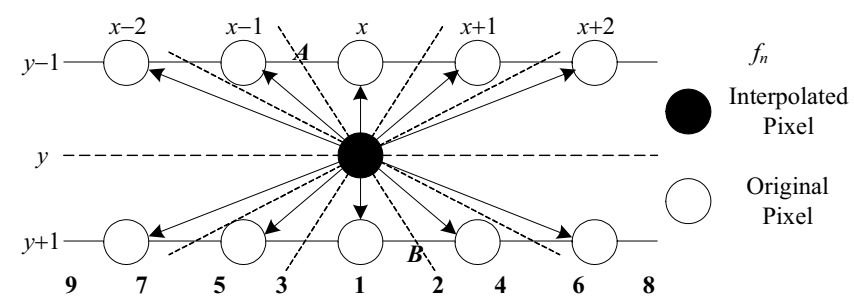

Fig. 4 Proposed EMF Algorithm in Sub-pixel Precision

If $\Delta_{x} A$ is less than 15 , we believe that there is no edge in position $A$. If no edge exists in position $B$ either, direction $A B$ will be excluded in the zigzag search. By the same token, we can deal with other sub-pixel directions.

Let $A e$ and $B e$ denote the values of the above and below pixels in the edge direction, respectively. The output of our EMF algorithm can be defined as:

$$
E M F_{n}(x, y)=\operatorname{Median}\left(\frac{A e+B e}{2}, \frac{u p+\text { down }}{2}, C, D, \frac{C+D}{2}\right),
$$

where up, down, $C$, and $D$ represent the spatially above, spatially below, forward referenced and backward referenced pixels, respectively. Note that the values of $C$ and $D$ depend on the motion vector selected in the last 
subsection. Equation (4) makes sense since the right hand side results in the same value as in motion trajectory, if the motion vector is reliable enough.

We have defined four possible moving states in [3], i.e. stationary, horizontally moving, vertically moving, and undirectionally moving. For the first three states, the deinterlacing scheme remains the same. For the undirectionally moving state, the proposed EMF is needed to reduce the impact of error propagations caused by the Adaptive Recursive (AR) [5] approach. If the texture is smooth, we simply use a linear interpolation, e.g. line averaging (LA); otherwise the deinterlacing output $F_{n}(x, y)$ should be defined as:

$$
F_{n}(x, y)=\beta \cdot A R_{n}(x, y)+(1-\beta) \cdot E M F_{n}(x, y),
$$

where $\beta$ satisfies the following equation:

$$
\beta=\operatorname{clip}\left(0,1, \operatorname{clip}\left(0,1, M V_{n}(x, y)-4\right)\right),
$$

with $M V_{n}(x, y)$ the absolute value of motion vector and $A R_{n}$ the output of AR. The clip function is defined as follows:

$$
\operatorname{clip}(a, b, c)=\left\{\begin{array}{l}
a, \text { if } c<a \\
b, \text { else if } c>b . \\
c, \text { otherwise }
\end{array}\right.
$$

\section{EXPERIMENTAL RESULTS}

\subsection{Performance of Proposed EMF and MVR}

Objectively well-performed EMF algorithms usually cannot result in excellent visual quality. Moreover, EMF is just proposed as one part of our adaptive deinterlacing in this paper. Hence it does not make much sense to compare the subjective results between our EMF and conventional EMF approaches, although actually our results are not worse.

In order to evaluate the objective performance of the proposed EMF and MVR, we extract interlaced videos from progressive ones by dropping one field in each frame. 16 progressive sequences with various resolutions are selected to generate interlaced videos. PSNR comparisons between original progressive sequences and deinterlaced videos are shown in Table 1, where one of the objectively best conventional EMF algorithms is provided as anchor.

It is easy to see that the proposed EMF is very efficient, as well as the MVR criterion. From the last two columns, we can also see their validity for adaptive deinterlacing.

\subsection{Performance of the New Adaptive Deinterlacing}

In [3] we have already shown that our adaptive deinterlacing scheme has better objective performance than conventional deinterlacing methods. Therefore it is unnecessary for us to compare the objective performance again since it performs even better than the former adaptive one, as can be seen from the last two columns of Table 1 . Fig. 5 shows the subjective effects on SD sequence "Basketball". Note that we successfully combine the advantages of LA and the proposed EMF while reducing the artifacts that caused by each of them.

Table 1. Objective Performance of Proposed EMF and MVR

\begin{tabular}{|c|c|c|c|c|c|c|c|}
\hline Sequence & Definition & $\begin{array}{c}\text { Number } \\
\text { of } \\
\text { Frames }\end{array}$ & $\begin{array}{c}\text { Conventional } \\
\text { EMF [3] }\end{array}$ & $\begin{array}{c}\text { Proposed } \\
\text { EMF }\end{array}$ & $\begin{array}{c}\text { Proposed } \\
\text { EMF } \\
\text { with } \\
\text { MVR }\end{array}$ & $\begin{array}{c}\text { Adaptive } \\
\text { Deinterlacing } \\
\text { with } \\
\text { Conventional } \\
\text { EMF [3] }\end{array}$ & $\begin{array}{c}\text { Adaptive } \\
\text { Deinterlacing } \\
\text { with Proposed } \\
\text { EMF and MVR }\end{array}$ \\
\hline News & CIF & 150 & 40.2 & 41.09 & 41.21 & 42.02 & 42.57 \\
\hline Bus & CIF & 150 & 24.48 & 24.94 & 25.5 & 26.92 & 27.62 \\
\hline Football & CIF & 150 & 27.15 & 27.42 & 27.53 & 32.16 & 32.72 \\
\hline Mobile & CIF & 150 & 26.46 & 27.57 & 28 & 26.94 & 27.79 \\
\hline Paris & CIF & 150 & 35.31 & 36.2 & 36.29 & 36.44 & 36.99 \\
\hline Tempete & CIF & 150 & 31.51 & 32.24 & 32.36 & 32.91 & 33.54 \\
\hline Crew & SD & 100 & 38.54 & 38.92 & 39.01 & 39.98 & 40.05 \\
\hline Night & SD & 100 & 34.48 & 34.79 & 34.97 & 36.02 & 36.2 \\
\hline City & HD & 50 & 32.42 & 32.89 & 33.08 & 33.37 & 33.74 \\
\hline Cyclists & HD & 50 & 39.23 & 39.86 & 40.06 & 40.54 & 41.04 \\
\hline Harbour & HD & 50 & 34.35 & 35.44 & 35.71 & 36.93 & 37.92 \\
\hline Night & HD & 50 & 33.19 & 33.33 & 33.66 & 35.99 & 36.12 \\
\hline Optis & HD & 50 & 40.68 & 40.99 & 41.13 & 40.12 & 40.63 \\
\hline Raven & HD & 50 & 38.35 & 38.72 & 39.38 & 42.19 & 42.6 \\
\hline Sheriff & HD & 50 & 39.61 & 39.96 & 40.01 & 39.46 & 39.7 \\
\hline Spin Calendar & HD & 50 & 28.23 & 28.28 & 28.39 & 29.59 & 29.98 \\
\hline
\end{tabular}




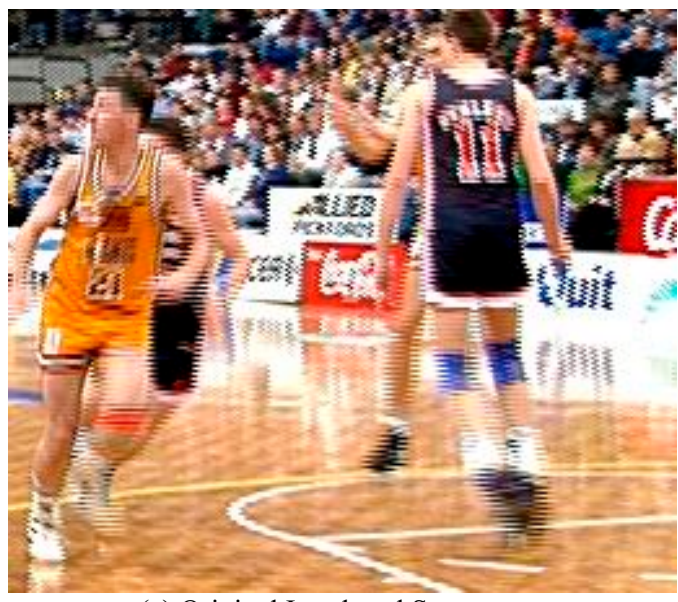

(a) Original Interlaced Sequence

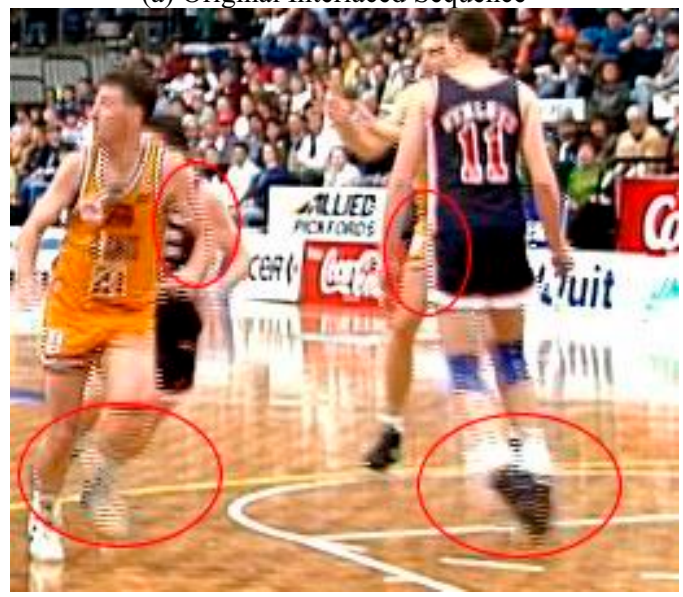

(c) Proposed EMF with MVR Analysis

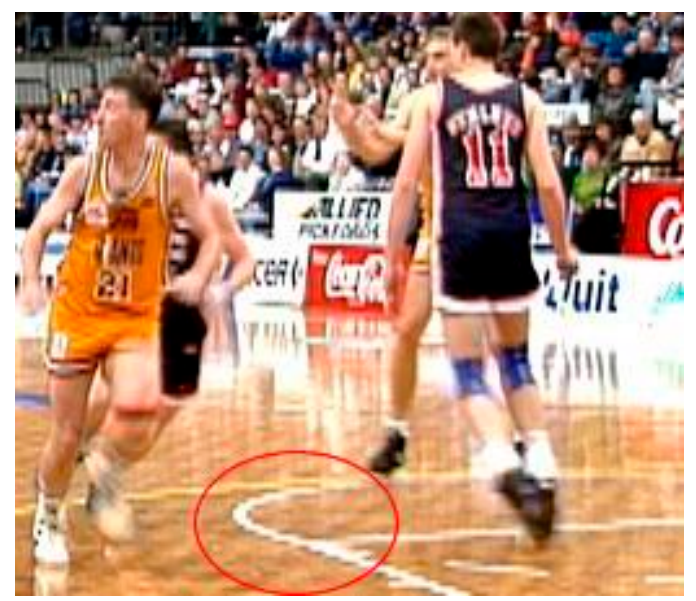

(b) LA

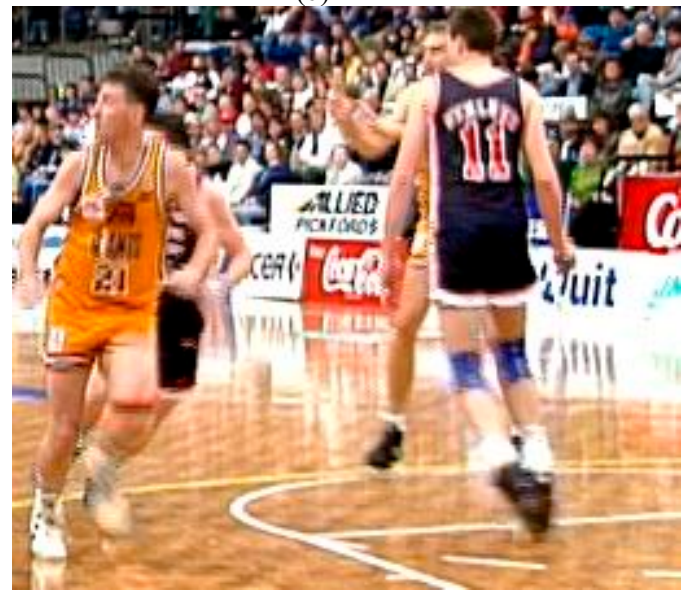

(d) Adaptive Deinterlacing with Proposed EMF \& MVR

Fig. 5 Subjective Deinterlacing Effects on SD Sequence "Basketball"

\section{CONCLUDING REMARKS AND FUTURE WORK}

In this paper we first introduce a criterion of motion vector reliability (MVR) for better deinterlacing along motion trajectory. Then we propose an edge-based median filtering (EMF) algorithm to improve objective performance while keeping at least the same subjective quality as objectively best EMF approaches nowadays. Experimental results on 16 sequences show that the proposed MVR and EMF are both efficient. We achieve significant PSNR improvement and at least the same subjective quality. The proposed EMF and MVR criterion are also valid for adaptive deinterlacing.

Since the MVR criterion is validated, a history-based model of motion detection will be studied in the next step to enhance the performance of the directional adaptive motion detection (DAMD) proposed in [3].

\section{ACKNOWLEDGMENT}

This work is partially supported by the National Natural Science Foundation of China under grant No. 60333020 and the Natural Science Foundation of Beijing under grant
No. 4041003. The authors would like to thank Dr. Cliff Reader and Qiang Wang for their helpful discussions.

\section{REFERENCES}

[1] N. C. Gallagher and G. L. Wise, "A theoretical analysis of the properties of median filters," IEEE Trans. Acoust., Speech, Signal Process., vol. 29, no. 6, pp. 1136-1141, Dec. 1981.

[2] G. de Haan and E. B. Bellers, "Deinterlacing - an overview," Proc. IEEE, vol. 86, no. 9, pp. 1839-1857, Sept. 1998.

[3] Q. Huang, W. Gao, D. Zhao, and H. Sun, "Adaptive deinterlacing for real-time applications," Proc. Pacific-Rim Conf. Multimedia, LNCS3768, Springer-Verlag, 2005, pp. 550-560.

[4] D. Wang, A. Vincent, and P. Blanchfield, "Hybrid deinterlacing algorithm based on motion vector reliability," IEEE Trans. Circuits Syst. Video Technol., vol. 15, no. 8, pp. 1019-1025, Aug. 2005.

[5] G. de Haan and E. B. Bellers, "Deinterlacing of video data," IEEE Trans. Consum. Electron., vol. 43, no. 3, pp. 819-825, Aug. 1997. 